\title{
PYRIMIDINE METABOLISM IN MAN. III. STUDIES ON LEUKOCYTES AND ERYTHROCYTES IN PERNICIOUS ANEMIA* $\dagger$
}

\author{
By LLOYD H. SMITH, JR. AND FAITH A. BAKER
}

\author{
(From the Department of Medicine, Massachusetts General Hospital, and Harvard Medical \\ School, Boston, Mass.)
}

(Submitted for publication July 30, 1959; accepted August 30, 1959)

The mechanism of action of vitamin $\mathrm{B}_{12}$ has not been established at a biochemical level. Evidence from both animal and microbiological studies has suggested that $B_{12}$ may serve as a cofactor in the neogenesis of methyl groups or in the formation of deoxyribose $(1,2)$. In such a capacity, $\mathrm{B}_{12}$ might be concerned with the synthesis of the methyl group of thymine (3) or of the pentose moiety of thymidylic and deoxycytidylic acids. In vitro studies of pernicous anemia marrow have indicated a direct stimulation by $\mathrm{B}_{12}$ of the incorporation of $\mathrm{C}^{14}$-formate into thymine (4). The partial remission in pernicious anemia produced by certain administered pyrimidines, notably orotic acid (5), supports the hypothesis that $\mathrm{B}_{12}$ deficiency leads to a derangement of pyrimidine metabolism in man. Direct analyses of marrow components in pernicious anemia have demonstrated abnormal relationships of nucleic acids such that the ratio of ribonucleic acid (RNA) to deoxyribonucleic acid (DNA) is increased (6). This report concerns studies of the cellular levels of three enzymes in pyrimidine biosynthesis using circulating erythrocytes and leukocytes from patients with pernicious anemia.

\section{EXPERIMENTAL PROCEDURE}

1. Enzymatic studies. Assays were carried out on aspartate carbamyltransferase, dihydroorotase and dihydroorotic dehydrogenase (Figure 1). The methods employed in separation of cells, sonication of leukocytes, hemolysis of erythrocytes and assay of the enzymes have been described in detail previously (7). In brief, each assay depended on the incorporation of a $\mathrm{C}_{14}$ precursor of high specific activity into a product which was then isolated

* This study was supported by Research Grants A-1606 and A-1606(C2) from the Institute of Arthritis and Metabolic Diseases, National Institutes of Health, United States Public Health Service, Bethesda, Md.

† A preliminary report of this work has been published in abstract form (Pyrimidine studies in pernicious anemia. J. clin. Invest. 1959, 38, 1044). and determined by the carrier technique. For comparative purposes results are expressed as millimicromoles ( $\mathrm{m} \mu \mathrm{Moles})$ of product synthesized per $10^{8}$ leukocytes or per $10^{\circ}$ erythrocytes.

2. Patients studied. Studies were carried out on 10 patients with pernicious anemia in relapse. (Incomplete studies were carried out on an additional patient.) The diagnoses were based on the cytological findings in blood and marrow and specific response to $B_{12}$ therapy. In some instances, the diagnosis was further supported by serum $B_{12}$ levels and positive Schilling tests. Hemoglobin levels averaged $6.3 \mathrm{Gm}$. per cent (range 3.7 to 8.5).

\section{RESULTS}

\section{Aspartate carbamyltransferase}

Aspartate carbamyltransferase, the first enzyme unique to pyrimidine biosynthesis, catalyzes the irreversible carbamylation of L-aspartic acid by carbamylphosphate to form carbamylaspartic acid (Figure 1) (8). A summary of the enzymatic activity of aspartate carbamyltransferase in normal erythrocytes and leukocytes and in the abnormal blood cells of 10 patients with pernicious anemia is presented in Figure 2A. Included for comparative purposes are studies of erythrocytes of one patient with the Di Guglielmo syndrome and the leukocytes of five patients with myelocytic leukemia. More detailed information concerning pyrimidine metabolism in leukemic cells is presented elsewhere (9). A considerable variation was found in cellular enzyme levels in pernicious anemia, but in both erythrocytes and leukocytes the activities were significantly greater than in normal cells $(p<0.01)$. Pernicious anemia erythrocytes averaged higher activities than those found in the immature erythrocytes of a patient with the Di Guglielmo syndrome with reticulocytosis (9 per cent) and circulating nucleated erythrocytes ( 55 per cent of nucleated cells). In some patients with pernicious anemia, the leukocyte aspartate carbamyltransferase was as elevated as that found in myelocytic leukemia. 
<smiles>NC(=O)[PH2+]C(N)C(N)C(=O)O</smiles>

ENZ. I - ASPARTATE CARBAMYLTRANSFERASE

ENZ.2 - DIHYDROOROTASE

ENZ.3 - DIHYDROOROTIC DEHYDROGENASE

Fig. 1. Pyrimidine Synthetic Enzymes Studied in Blood Cerls

CAP, carbamylphosphate; L-ASP, L-aspartic acid; CAA, carbamylaspartic acid; DHO, dihydroorotic acid; OA, orotic acid.

\section{Dihydroorotase}

Dihydroorotase catalyzes the reversible cyclization of carbamylaspartic acid to form dihydroorotic acid (Figure 1) (10). The biochemical characteristics of this enzyme in human blood cells have been described elsewhere (7). In Figure $2 \mathrm{~B}$ a summary is given of the enzymatic activities of dihydroorotase found in the erythrocytes and leukocytes of pernicious anemia in comparison with normal enzyme levels and in contrast to the values in the Di Guglielmo syndrome and myelocytic leukemia. Despite the variations in individual patients, the enzyme levels are again significantly increased $(p<0.01)$ in both erythrocytes and leukocytes. In several patients with pernicious anemia, leukocyte dihydroorotase was found to be within the range of myelocytic leukemia.

\section{Dihydroorotic dehydrogenase}

Dihydroorotic dehydrogenase catalyzes the reversible oxidation of dihydroorotic acid (DHO) to orotic acid (OA) as shown in Figure 1 (11). In previous studies this enzyme was found to be absent in mature erythrocytes and present in reproducible activity in leukocytes (7). By cell fractionation studies the enzymatic activity was found to be largely in the cell nucleus. In Figure $2 \mathrm{C}$ the levels of dihydroorotic dehydrogenase activity are summarized for pernicious anemia, normal cells, Di Guglielmo syndrome and myelocytic leukemia. In the erythrocytes of pernicious anemia, dihydroorotic dehydrogenase could not be detected, as it can be, in normal erythrocytes. In contrast, the abnormal nucleated erythrocytes of the Di Guglielmo syndrome exhibited this activity. In the leukocytes of pernicious anemia, dihydroorotic dehydrogenase activity was normal, except for one deviant value. This is in contrast to the marked increase of activity noted in leukemic cells.

\section{Reversibility of the enzyme abnormalities}

All of the patients studied responded hematologically to parenteral $\mathrm{B}_{12}$ therapy. In two of the patients, follow-up studies were carried out after hematological remission was complete. Enzymatic activities of aspartate carbamyltransferase and dihydroorotase returned to normal (Figure 3 ). The time course of this "enzymatic remission" was not followed because of the complications introduced by reticulocytosis and variations in cell age.

\section{Relationship of enzyme abnormalities to the se- verity of pernicious anemia}

An attempt was made to demonstrate some relationship between the increase of enzymatic activities and the severity of pernicious anemia, as indicated by the level of the initial hemoglobin. Although, in general, patients with the severest anemia seemed to have the greatest cellular enzymatic alterations, this relationship was not well defined. This is perhaps not surprising in view of the variation in the systemic response of patients to $B_{12}$ deficiency-variations in the relative de- 
gree of neurological, epithelial and hematological abnormalities.

\section{DISCUSSION}

Increased activities of aspartate carbamyltransferase and dihydroorotase were consistently found in both the erythrocytes and leukocytes of patients with pernicious anemia in relapse. There is no evidence from extensive animal and bacteriological studies that vitamin $B_{12}$ is directly concerned in the synthesis or action of these two enzymes (1, 12). The first possibility to be considered is that

\section{A}

\section{ASPARTATE CARBAMYLTRANSFERASE}

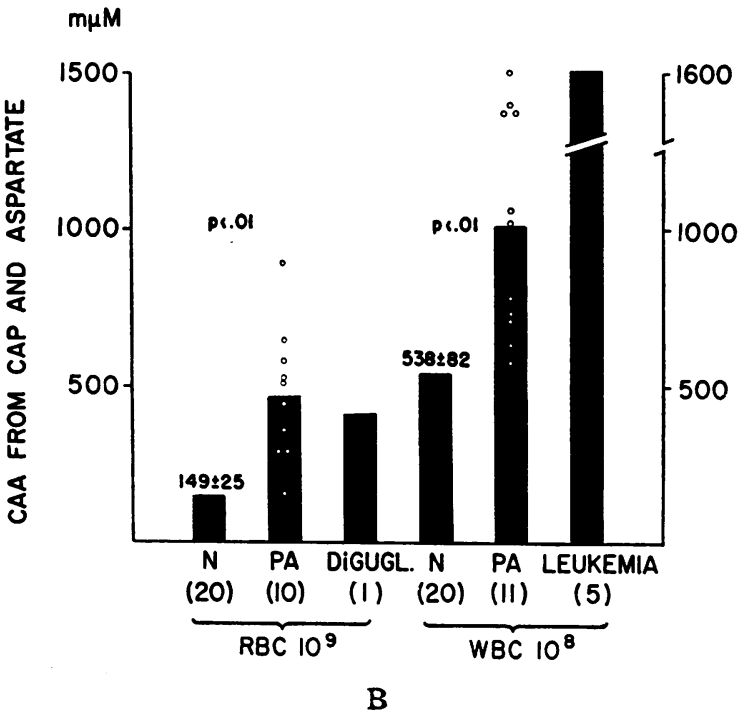

\section{DIHYDROOROTASE}

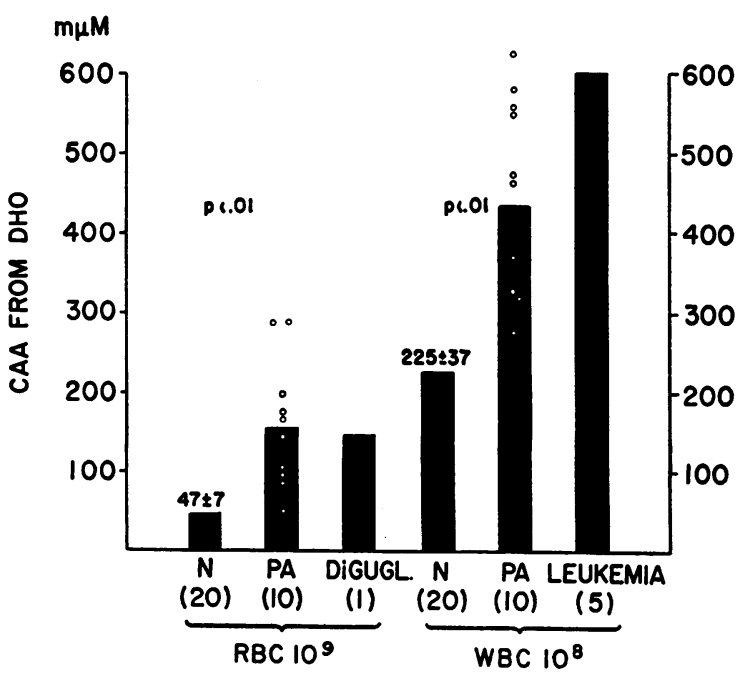

Fig. 2-Continued

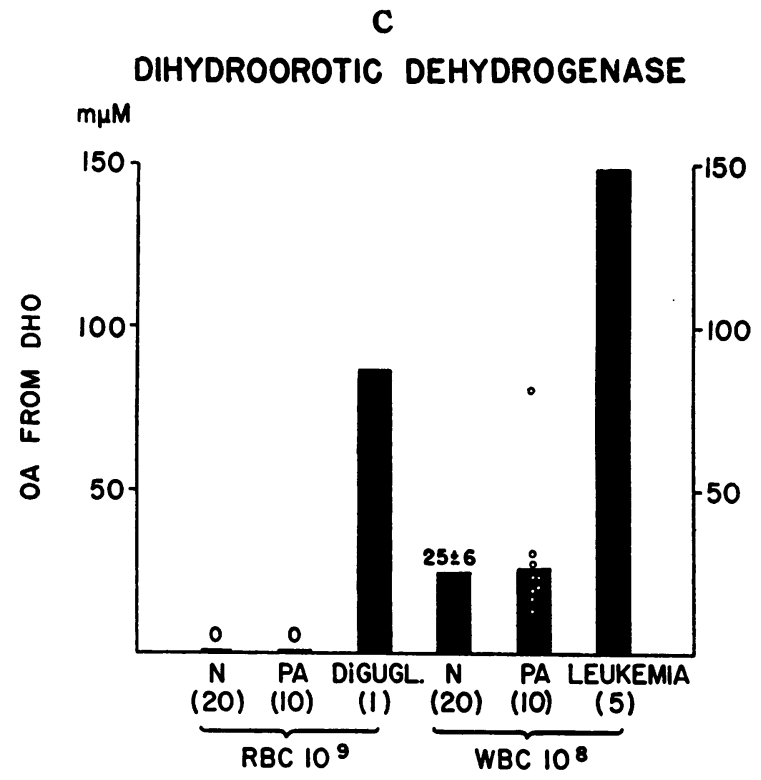

Fig. 2. Levels of Aspartate Carbamyltransferase (A) Dihydroorotase (B), and Dihydroorotic DehyDROGENASE (C), RESPECTIVELy, IN THE LeUKOCYTES AND Erythrocytes of Pernicious Anemia

These levels are compared with those found in normal cells, leukemia and the Di Guglielmo syndrome.

the enzymatic abnormalities found represent a nonspecific increase of enzyme levels in young cells. It has been clearly shown that pernicious anemia is, in part, a hemolytic anemia with increased destruction of erythrocytes in the peripheral blood and probably within the bone marrow (13). The circulating macrocyte is therefore a young cell. Evidence from several sources suggests that immaturity of cells does not account for the increase of enzyme activity observed. There is no cytological evidence of immaturity of circulating polymorphonuclear leukocytes in pernicious anemia. The enzymatic activities of aspartate carbamyltransferase and dihydroorotase observed were comparable to those found in the Di Guglielmo syndrome and myelocytic leukemia, conditions of much greater immaturity of cells as judged by cytological criteria. Finally, the pattern of alteration of enzyme activity in the leukocyte was not that found in immaturity per se. An increase of dihydroorotic dehydrogenase activity is the most striking abnormality of the immature leukocyte, as demonstrated in myelocytic leukemia and in the leukocytosis of infection (9). In pernicious anemia leukocyte dihydroorotic dehydrogenase ac- 
A

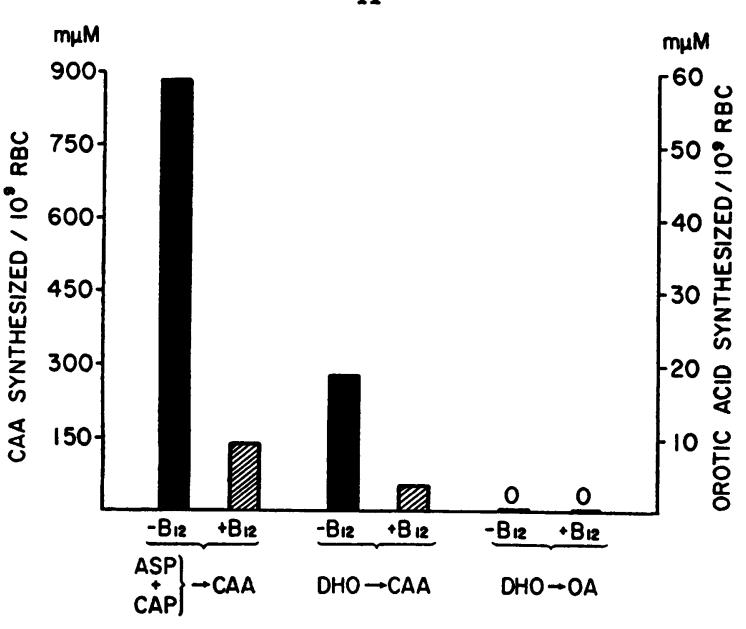

B

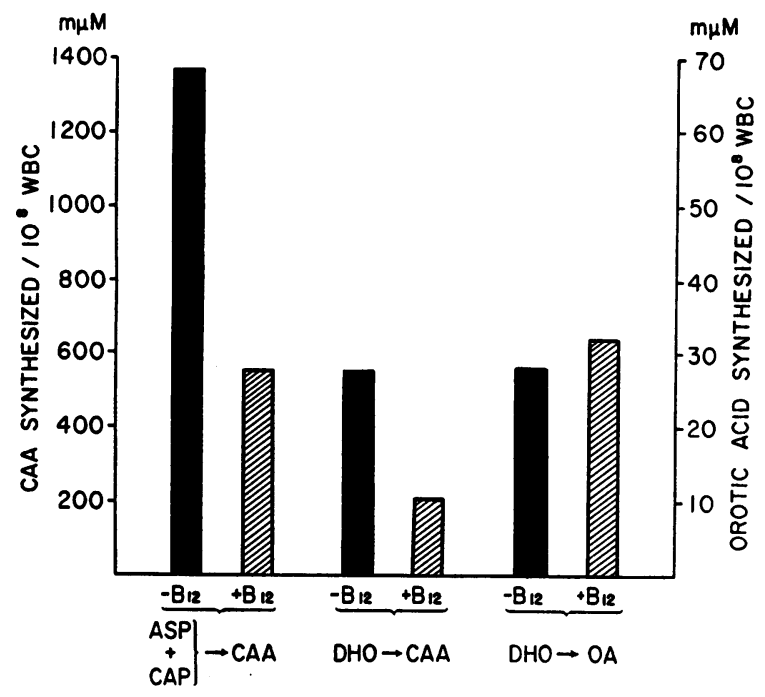

Fig. 3. Enzyme Levels in the Erythrocytes (A), and Leukocytes (B), Respectively, of a Patient with Pernicious Anemia Before and After Therapy with Vitamin $B_{12}$

Before treatment $\mathrm{Hgb}$. was $4.6 \mathrm{Gm}$. per cent; three months after treatment, $\mathrm{Hgb}$. was 12.4. ASP + CAP $\rightarrow$ CAA, aspartate carbamyltransferase; DHO $\rightarrow$ CAA, dihydroorotase; DHO $\rightarrow \mathrm{OA}$, dihydroorotic dehydrogenase.

tivity is normal. In brief, there is a consistent cellular abnormality of certain pyrimidine synthetic enzymes in pernicious anemia in a pattern different from that of immaturity of cells per se. The altered enzyme pattern returns to normal following treatment with vitamin $B_{12}$.

It would be beyond the scope of this discussion to review current theories of the mechanism of ac- tion of vitamin $B_{12}$. The function of $B_{12}$ appears to vary in different organisms. One of its most firmly established metabolic roles relates to the neogenesis of methyl groups in certain microorganisms (3) and animals (1). In this way $B_{12}$ might be concerned with the synthesis of thymine, which has been reported to produce a partial remission in pernicious anemia when given in large oral doses (14). In marrow cells from pernicious anemia studied in vitro, the addition of $\mathrm{B}_{12}$ to the plasma suspension has been shown to stimulate the incorporation of $\mathrm{C}^{14}$-formate into thymine, presumably an indication of increased synthesis of DNA (4). There is evidence that as a second function, $B_{12}$ may be concerned with the synthesis of deoxyriboside or deoxyribotide derivatives of purine and pyrimidine bases (2). Most of this work has been carried out on microorganisms. Partial remissions of pernicious anemia may be produced by a large amount of a number of pyrimidines (5). Whatever the exact function of $\mathrm{B}_{12}$ may be in man, there is evidence of a derangement in pyrimidine metabolism in blood cells in $B_{12}$ deficiency.

A major interest in current biochemistry is the study of methods by which enzymatic reactions are appropriately controlled within the cell (15). One of these methods of control is that of the negative feedback reaction. In the first type of negative feedback reaction, the product of a series of biochemical reactions controls, seemingly by inhibition, the production of the enzymes involved

\section{FEEDBACK CONTROL-I. (ENZYME CONCENTRATION)}

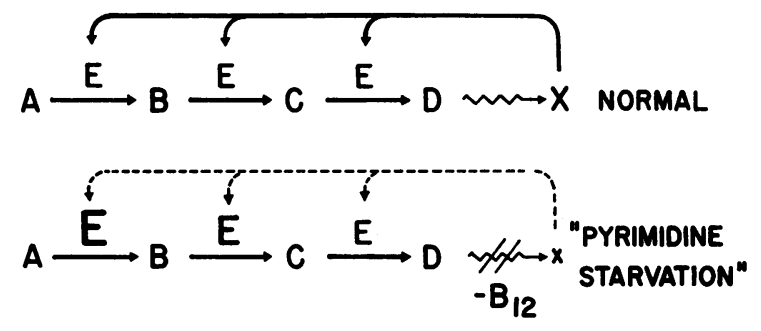

Fig. 4. Schematic Representation of the Negative Feedback Control of Proximal Enzyme Synthesis Exerted by the End Product (X) in a Sequence

With a block in the sequence (postulated here as due to $B_{12}$ deficiency) there is a release of inhibition and compensatory "hypertrophy" of the proximal enzymes. 
in its own biosynthesis (Figure 4). This controlling mechanism has been clearly shown to be operative in pyrimidine synthesis in bacteria by Yates and Pardee (16). When there is a genetic block in pyrimidine synthesis, bacterial growth continues only if a pyrimidine precursor distal to the missing enzyme is furnished in the medium. If this precursor is withdrawn, there is "pyrimidine starvation" with an accompanying increase of the enzymatic activities proximal to the blocked step. It has been suggested that a fall in the concentration of the end product releases an inhibition which governs the rate at which earlier enzymes are synthesized. The resulting increase in enzyme concentration would serve as a homeostatic mechanism to maintain the optimal rate of production of the end product. The pattern of increased enzyme activity in bacteria with a block in pyrimidine synthesis is: aspartate carbamyltransferase $>$ dihydroorotase $>$ dihydroorotic dehydrogenase. This is the pattern found in pernicious anemia, and is the obverse of that found in immaturity of blood cells. The current studies are interpreted as being consistent with the pattern of "pyrimidine starvation" in human blood cells, in which a partial block in pyrimidine synthesis occurs secondary to $\mathrm{B}_{12}$ deficiency rather than a complete block in Escherichia coli due to the genetic ablation of an enzyme.

A second type of negative feedback reaction has been described in pyrimidine synthesis in $E$. coli in which the product serves as a competitive inhibitor of the first enzyme in the biosynthetic sequence (Figure 5) (17). This is inhibition of enzyme activity rather than enzyme concentration. A similar controlling mechanism has also been described in purine synthesis (18). The inhibition of aspartate carbamyltransferase by cytidine- 5 'phosphate in $E$. coli was readily confirmed. Attempts to demonstrate this type of feedback control in human erythrocytes or leukocytes with either cytidine-5'-phosphate or uridine-5'-phosphate have been unsuccessful. In a patient with orotic aciduria large amounts of orotic acid appeared in the urine in association with refractory megaloblastic anemia (19). When a mixture of nucleotides was given there was a sharp reduction in orotic aciduria. This reduction in orotic acid synthesis was probably mediated by one or both

\section{FEEDBACK CONTROL-II. (ENZYME INHIBITION)}

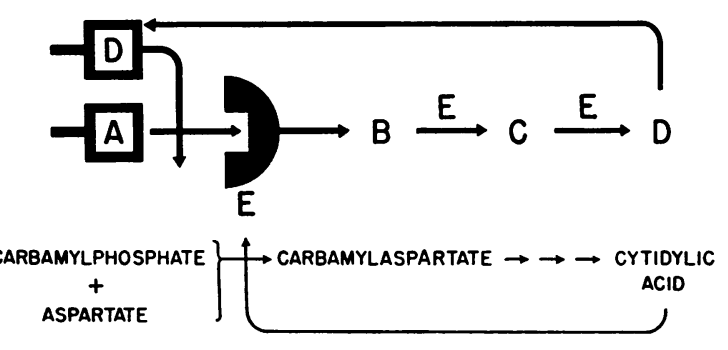

Fig. 5. Schematic Representation of a Second Negative Feedback Control Mechanism in Pyrimidine Synthesis, the Competitive Inhibition of the First Enzyme in the Sequence, Aspartate Carbamyltransferase, by a Later Product, Cytidine-5'-PhosPHATE

of the negative feedback control mechanisms outlined above.

\section{SUM MARY}

1. Three enzymes leading to the synthesis of orotic acid-aspartate carbamyltransferase, dihydroorotase and dihydroorotic dehydrogenase-were studied in the erythrocytes and leukocytes of 10 patients with pernicious anemia in relapse.

2. Increased activities of aspartate carbamyltransferase and dihydroorotase with normal activity of dihydroorotic dehydrogenase were found. This pattern differs from that found in immaturity of cells per se. The abnormal pattern returned to normal following treatment with vitamin $B_{12}$.

3. It is suggested that the enzyme abnormalities are most consistent with the pattern of "pyrimidine starvation" produced by vitamin $\mathrm{B}_{12}$ deficiency, the indirect evidence of a negative feedback control of enzyme concentrations in blood cells.

4. A second negative feedback control mechanism, the competitive inhibition of aspartate carbamyltransferase by cytidine- 5 '-phosphate, was confirmed in Escherichia coli but could not be demonstrated in erythrocytes or leukocytes.

\section{REFERENCES}

1. Johnson, B. C. Studies on the mechanism of action of vitamin $B_{12}$ in animal nutrition. Amer. J. clin. Nutr. 1958, 6, 34.

2. Downing, M., and Schweigert, B. S. Role of vita$\min B_{12}$ in nucleic acid metabolism. IV. Metabo- 
lism of $\mathrm{C}^{14}$-labelled thymidine by Lactobacillus leichmanii. J. biol. Chem. 1956, 220, 521.

3. Dinning, J. S., Allen, B. K., Young, R. S., and Day, $P$. L. The role of vitamin $B_{12}$ in thymine biosynthesis by Lactobacillus leichmanii. J. biol. Chem. 1958, 233, 674.

4. Thomas, E. D., and Lochte, H. L., Jr. Studies on the biochemical defect of pernicious anemia. I. In vitro observations on oxygen consumption, heme synthesis, and deoxyribonucleic acid synthesis by pernicious anemia bone marrow. J. clin. Invest. 1958, 37, 166.

5. Rundles, R. W., and Brewer, S. S., Jr. Hematologic responses in pernicious anemia to orotic acid. Blood 1958, 13, 99.

6. Glazer, H. S., Mueller, J. F., Jarrold, T., Sukurai, K., Will, J. J., and Vilter, R. W. The effect of vitamin $B_{12}$ and folic acid on nucleic acid composition of the bone marrow of patients with megaloblastic anemia. J. Lab. clin. Med. 1954, 43, 905.

7. Smith, L. H., Jr., and Baker, F. A. Pyrimidine metabolism in man. I. The biosynthesis of orotic acid. J. clin. Invest. 1959, 38, 798.

8. Lowenstein, J. M., and Cohen, P. P. Studies on the mechanism of carbamylaspartic acid synthesis. J. biol. Chem. 1955, 213, 689.

9. Smith, L. H., Jr., and Baker, F. A. Pyrimidine metabolism in man. II. Studies in leukemia. Blood. In press.

10. Lieberman, I., and Kornberg, A. Enzymatic synthesis and breakdown of a pyrimidine, orotic acid. II. Dihydroorotic acid, ureidosuccinic acid, and 5-carboxymethylhydantoin. J. biol. Chem. 1954, 207, 911.
11. Lieberman, I., and Kornberg, A. Enzymatic synthesis and breakdown of a pyrimidine, orotic acid. I. Dihydro-orotic dehydrogenase. Biochim. biophys. Acta 1953, 12, 223.

12. Williams, R. T., Ed. The Biochemistry of Vitamin $\mathrm{B}_{12}$. Biochemical Society Symposia No. 13, London, Cambridge Univ. Press, 1955.

13. Finch, C. A., Coleman, D. H., Motulsky, A. G., Donohue, D. M., and Reiff, R. H. Erythrokinetics in pernicious anemia. Blood 1956, 11, 807.

14. Vilter, R. W., Horrigan, D., Mueller, J. F., Jarrold, T., Vilter, C. F., Hawkins, V., and Seaman, A. Studies on the relationships of vitamin $B_{12}$, folic acid, thymine, uracil, and methyl group donors in persons with pernicious anemia and related megaloblastic anemias. Blood 1950, 5, 695 .

15. Pardee, A. B. The control of enzyme activity in The Enzymes, 2nd ed. New York, Academic Press Inc., 1959, pp. 681-716.

16. Yates, R. A., and Pardee, A. B. Control by uracil of formation of enzymes required for orotate synthesis. J. biol. Chem. 1957, 227, 677.

17. Yates, R. A., and Pardee, A. B. Control of pyrimidine biosynthesis in Escherichia coli by a feed-back mechanism. J. biol. Chem. 1956, 221, 757.

18. Wyngaarden, J. B., and Ashton, D. M. The regulation of activity of phosphoribosylpyrophosphate amidotransferase by purine ribonucleotides: A potential feedback control of purine biosynthesis. J. biol. Chem. 1959, 234, 1492.

19. Huguley, C. M., Jr., Bain, J. A., Rivers, S. L., and Scoggins, R. B. Refractory megaloblastic anemia associated with excretion of orotic acid. Blood $1959,14,615$.

\section{ANNOUNCEMENT}

A Grant Award for travel funds to the First International Congress of Nephrology in Geneva, Switzerland, September 1, 2, 3, 1960, has been awarded by the U.S.P.H.S. to the American Society for Clinical Investigation.

Members and nonmembers are eligible to apply for travel funds to the Congress. Funds are available to send 20 individuals to the meetings.

Applications for funds should include in quadruplicate:

a) Letter of application stating interest in the field and in the Congress.

b) Curriculum vitae.

The deadline for receipt of $a$ ) and $b$ ) is March 1, 1960. Awards will be made around March 15, 1960.

Applications should be sent to Saul J. Farber, Secretary, American Society for Clinical Investigation, New York University College of Medicine, 550 First Avenue, New York 16, New York. 